\section{PTH-145 VALIDATION OF DIRECT OBSERVATION OF PROCEDURAL SKILLS (DOPS) ASSESSMENTS FOR PAEDIATRIC GASTROSCOPY}

${ }^{1}$ Keith Siau*, ${ }^{2}$ Rachel Levi, ${ }^{2}$ Lucy Howarth, ${ }^{1}$ Raphael Broughton, ${ }^{2}$ Christos Tzivinokos, ${ }^{1}$ Mark Feeney, ${ }^{2}$ Priya Narula. ${ }^{1}$ Joint Advisory Group, London, UK ${ }^{2} B S P G H A N$, UK

\subsection{6/gutjpl-2018-BSGAbstracts.544}

Introduction Direct observation of procedural skills (DOPS) are tools designed by the Joint Advisory Group (JAG) to assess competence in endoscopy. These were expanded in July 2016 (new DOPS) to include those specific to paediatric gastroscopy (OGD). However, paediatric OGD DOPS assessments have not been validated.

Methods We performed a prospective UK-wide analysis of formative paediatric OGD DOPS submitted to the JETS e-Portfolio over one-year (August 2016-2017). Scores were averaged across procedural domains (pre-procedural, procedural, postprocedural and endoscopic non-technical skills - ENTS), and according to skillset (cognitive or technical), and compared with the overall performance score using Spearman's correlation. Overall scores were also compared by trainee grade.

Results 157 DOPS assessments were completed by 20 unique trainers for 17 trainees. Overall performance score comprised: 1: Maximal supervision (4.5\%), 2: Significant supervision (14.0\%), 3: Minimal supervision (24.8\%) and 4: Competent (56.7\%). By domain, overall competence correlated most with mean scores for the 'Insertion and Withdrawal' domain (rho: 0.884, p<0.001), Management (rho 0.834, p<0.001), Visualisation (rho $0.819, \mathrm{p}<0.001)$, ENTS $(0.773, \mathrm{p}<0.001$ ), 'Postprocedural' (rho 0.611, $\mathrm{p}<0.001$ ) and pre-procedural (rho $0.575, \mathrm{p}<0.001)$. By skillset, overall score correlated most with performance in 'Technical' (rho 0.860, p $<0.001$ ), followed by ENTS and 'Cognitive' domains (rho 0.742, $\mathrm{p}<0.001)$ domains compared to ENTS. In terms of DOPS items, overall competence score correlated most with 'Management of Complications' (rho 0.852, p<0.001) and 'Angulation and Tip Control' (rho 0.834, p<0.001), and least with 'Confirms Consent' (rho 0.396, p<0.001) and 'Equipment Check' (rho $0.528, \mathrm{p}<0.001$ ). Overall scores correlated with seniority of training $(\mathrm{p}<0.001)$ [figure 1], demonstrating construct validity.

Conclusions Competencies in paediatric OGD, as assessed within DOPS, vary in their correlation with overall competence. Strong correlations were reported for the 'Insertion and Withdrawal' domain, 'Management of Complications' items, and 'Technical' skillsets. As assessors are completing the new DOPS in a consistent manner, with scores improving by seniority, this provides validity evidence for the new paediatric OGD DOPS.

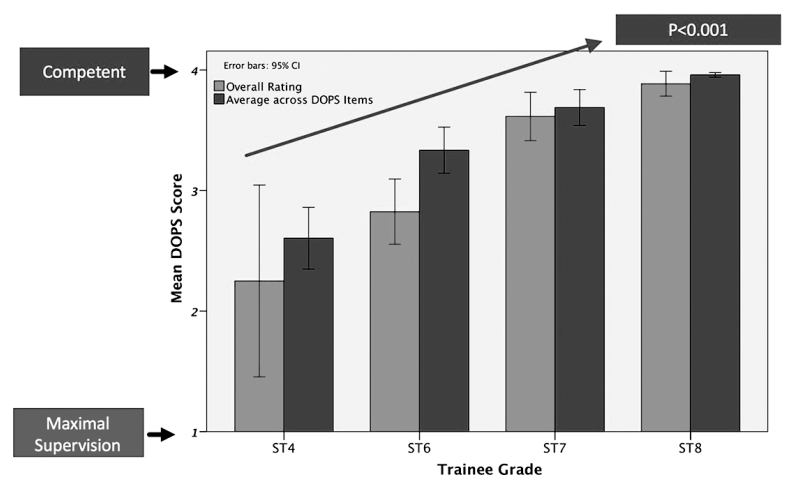

Abstract PTH-145 Figure 1

\section{PTH-146 VALIDATION OF DIRECT OBSERVATION OF PROCEDURAL SKILLS (DOPS) ASSESSMENTS FOR PAEDIATRIC COLONOSCOPY}

${ }^{1}$ Keith Siau*, ${ }^{2}$ Rachel Levi, ${ }^{2}$ Lucy Howarth, ${ }^{1}$ Raphael Broughton, ${ }^{2}$ Christos Tzvinokos, ${ }^{1}$ Mark Feeney, ${ }^{2}$ Priya Narula. ${ }^{1}$ Joint Advisory Group, London, UK; ${ }^{2} B S P G H A N$, UK

\subsection{6/gutjnl-2018-BSGAbstracts.545}

Introduction Direct observation of procedural skills (DOPS) are tools designed by the Joint Advisory Group (JAG) to assess competence in endoscopy. These were expanded in July 2016 (new DOPS) to include those specific to paediatric colonoscopy. However, paediatric colonoscopy DOPS assessments have not been validated. We aimed to correlate overall trainee competence with components of the paediatric colonoscopy DOPS.

Subjects and Methods We performed a prospective UK-wide analysis of formative paediatric colonoscopy DOPS submitted to the JETS e-Portfolio over one-year (August 2016-2017). Scores were averaged across procedural domains (pre-procedural, procedural, post-procedural and endoscopic non-technical skills - ENTS). Each DOPS item, except for ENTS, were grouped into cognitive and technical skillsets by two independent investigators, and correlated with the overall performance score. Correlation analyses were performed using Spearman's test (rho $>0.70$ indicating high positive correlation).

Results 61 DOPS assessments were completed by 13 unique trainers for 14 trainees. Overall performance score comprised: 1: Maximal supervision (1.6\%), 2: Significant supervision (13.1\%), 3: Minimal supervision (47.5\%) and 4: Competent (37.7\%). By domain, overall competence correlated most with scores for the 'Procedural' domain (rho: 0.849, $\mathrm{p}<0.001$ ), ENTS (0.666, p<0.001), 'Post-procedural' (rho 0.635, $\mathrm{p}<0.001$ ) and pre-procedural (rho 0.471, p<0.001). By domain, overall score correlated more with performance in predominantly 'Cognitive' (rho 0.834, p<0.001) and 'Technical' (rho 0.815, p<0.001) domains compared to ENTS. In terms of DOPS items, overall competence score correlated most with 'Proactive Problem Solving' (rho 0.836, p<0.001) and 'Patient Comfort' (rho 0.826, p<0.001), and weakest with 'Confirms Consent' (rho 0.228, p=0.115) and 'Equipment Check' (rho 0.302, p=0.020).

Conclusion Competencies in paediatric colonoscopy, as assessed within DOPS, vary in their correlation with overall competence. Performance in the 'Procedural' domain, Proactive Problem Solving' items, and 'Cognitive' skillsets had greatest correlation with overall procedural competence. As assessors are completing the new DOPS in a consistent manner, this provides novel validity evidence for the new paediatric colonoscopy DOPS.

\section{PTH-147 IMPROVING JUNIOR DOCTORS CONFIDENCE AND COMPETENCE IN CONSENTING PATIENTS FOR ERCP - AN EDUCATIONAL INTERVENTION}

${ }^{1}$ Heather Walker*, ${ }^{1}$ Heather Cracknell, ${ }^{1}$ Hannah Dix, ${ }^{1}$ Amin Elshibly, ${ }^{2}$ Mark Smith, ${ }^{1}$ Maximillian Groome. ${ }^{1}$ Gastroenterology Department, Ninewells Hospital, Dundee, UK ${ }^{2}$ Dundee Institute for Healthcare Simulation, University of Dundee, Dundee, UK

\subsection{6/gutjnl-2018-BSGAbstracts.546}

Introduction Obtaining high quality informed consent for endoscopic procedures is frequently devolved to Junior Doctors, within NHS Tayside there was previously no standardised 